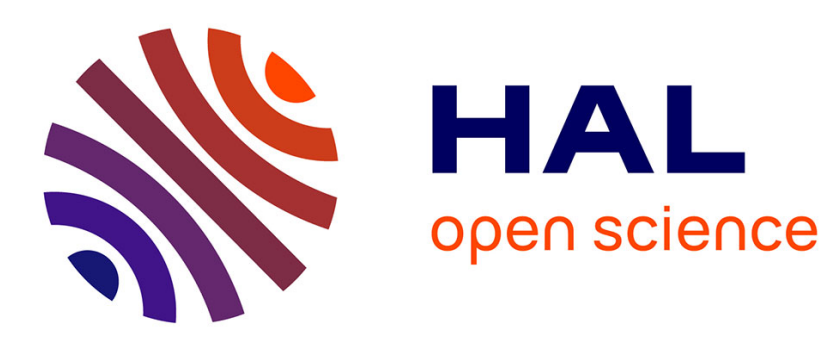

\title{
Equilibrium Shapes of Large Trans-Neptunian Objects
}

Nicolas Rambaux, Daniel Baguet, Fréderic Chambat, Julie Castillo-Rogez

\section{To cite this version:}

Nicolas Rambaux, Daniel Baguet, Fréderic Chambat, Julie Castillo-Rogez. Equilibrium Shapes of Large Trans-Neptunian Objects. The Astrophysical journal letters, 2017, 850 (1), pp.L9. 10.3847/2041-8213/aa95bd . hal-01662024

\section{HAL Id: hal-01662024 \\ https://hal.sorbonne-universite.fr/hal-01662024}

Submitted on 12 Dec 2017

HAL is a multi-disciplinary open access archive for the deposit and dissemination of scientific research documents, whether they are published or not. The documents may come from teaching and research institutions in France or abroad, or from public or private research centers.
L'archive ouverte pluridisciplinaire HAL, est destinée au dépôt et à la diffusion de documents scientifiques de niveau recherche, publiés ou non, émanant des établissements d'enseignement et de recherche français ou étrangers, des laboratoires publics ou privés. 
Draft VERSion November 7, 2017

Typeset using $\mathrm{LAT}_{\mathrm{E}} \mathrm{X}$ default style in AASTeX61

\title{
EQUILIBRIUM SHAPES OF LARGE TRANSNEPTUNIAN OBJECTS
}

\author{
Nicolas Rambaux, ${ }^{1}$ Daniel Baguet,${ }^{2}$ Frederic Chambat ${ }^{3}$ And Julie C. Castillo-Rogez ${ }^{4}$
}

${ }^{1}$ IMCCE, Observatoire de ParisPSL Research University, Sorbonne UniversitésUPMC Université Paris 06, Université Lille 1, CNRS, 77 avenue Denfert-Rochereau, 75014 Paris, France

2 Institut UTINAM UMR6213, CNRS, Univ. Bourgogne Franche-Comté, OSU Theta F-25000 Besançon, France

${ }^{3}$ LGLTPE, CNRS UMR5276, ENS de Lyon, Site Monod, 15 parvis René Descartes; Lyon, F-69007, France

${ }^{4}$ Jet Propulsion Laboratory, California Institute of Technology, 4800 Oak Grove Drive, Pasadena, 91109 CA, United States

(Received November 7, 2017; Revised XX; Accepted XX)

\section{Submitted to ApJL}

\begin{abstract}
The large transneptunian objects (TNO) with radii larger than $400 \mathrm{~km}$ are thought to be in hydrostatic equilibrium. Their shapes can bring clues on their internal structures that would reveal information on their formation and evolution. In this paper we explore the equilibrium figures of five TNOs and we show that the difference between the equilibrium figures of homogeneous and heterogeneous interior models can reach several kilometers for fast rotating and low density bodies. Such a difference could be measurable by ground-based techniques. This demonstrates the importance of developing the shape up to second and third order when modeling the shapes of large and rapid rotators.
\end{abstract}

ABSTRACT

Keywords: minor planets, asteroids: general - methods: numerical - gravitation 
Table 1. Physical properties of the selected TNO. (a) Brown (2013); (b) Heinze \& de Lahunta (2009); (c) Fornasier et al. (2013); (d) Thirouin et al. (2010); (e) Ortiz et al. (2003); (f) Braga-Ribas et al. (2013); (g) Gaudi et al. (2005); (h) Thirouin et al. (2014); $\left(^{*}\right)$ preferred period of Thirouin et al. (2010) and (**) preferred period of Thirouin et al. (2014).

\begin{tabular}{|c|c|c|c|c|c|}
\hline Bodies - Parameters & $\begin{array}{r}\text { Equivalent } \\
\text { Radius }(\mathrm{km})\end{array}$ & $\begin{array}{r}\text { Mean density } \\
\left(\mathrm{kg} / \mathrm{m}^{3}\right) \\
\end{array}$ & $\begin{array}{r}\text { Rotation Period } \\
\text { (hours) }\end{array}$ & $\begin{array}{l}\text { Rotation ratio } \\
\qquad \frac{\Omega^{2}}{\pi G \rho} \\
\end{array}$ & References \\
\hline (136472) Makemake & $715 \pm 3.5$ & - & $7.771 \pm 0.003$ & - & $(\mathrm{a}, \mathrm{b})$ \\
\hline \multirow[t]{3}{*}{ (50000) Quaoar } & $535 \pm 19$ & $2180_{-360}^{+430}$ & $8.84 \pm 0.01$ & 0.085 & $(\mathrm{c}, \mathrm{d})$ \\
\hline & & & $17.6788 \pm 0.0004$ & & $(\mathrm{c}, \mathrm{e})$ \\
\hline & $555 \pm 2.5$ & $1990 \pm 460$ & $8.84 \pm 0.01$ & 0.093 & $(\mathrm{f}, \mathrm{d})$ \\
\hline (90377) Sedna & $497.5 \pm 40$ & - & $10.273 \pm 0.002$ & - & $(\mathrm{g})$ \\
\hline (90482) Orcus & $458.5 \pm 12.5$ & $1530_{-130}^{+150}$ & $10.47^{(*)}$ & 0.087 & $(\mathrm{c}, \mathrm{d})$ \\
\hline (120347) Salacia & $427 \pm 22.5$ & $1290_{-230}^{+290}$ & $6.5^{(* *)}$ & 0.267 & $(\mathrm{c}, \mathrm{h})$ \\
\hline
\end{tabular}

\section{INTRODUCTION}

Dwarf planets and transneptunian objects (TNO) with radii larger than $400 \mathrm{~km}$ should present a spheroidal shape corresponding to hydrostatic equilibrium but the exact limit radius at which "the self-gravity overcome rigid tidal forces" as defined by IAU is still debated (e.g. Tancredi \& Favre 2008). That limit radius depends on composition but also on the heat budget of the body. A body may be large enough to differentiate and to relax into hydrostatic equilibrium, but it might not benefit from enough heat, over the long-term, for its shape to recover hydrostatic equilibrium following large collisional events. In this study we investigate five ice-rich bodies TNOs with a radius larger than $400 \mathrm{~km}$ and a short spin period, between 6 hours and 13 hours. We assume that these bodies are relaxed to hydrostatic equilibrium and some of their measured and deduced characteristics are listed in Table 1. The list includes KBOs (Kuiper Belt Objects): Makemake, Quaoar, Orcus, Salacia, and the inner Oort cloud object Sedna. We assess the influence of internal stratification on the equilibrium figure of these fast rotators, assuming two-layer models composed of ice and silicate. To first order, the ice-silicate ratio informs on the formation and evolution of these large planetesimals (Barr \& Schwamb 2016).

The hydrostatic equilibrium figure of a differentiated body can be determined by solving numerically the Clairaut's equations developed to an order that depends on the geodetic parameter $m$ (see Chambat et al. 2010; Rambaux et al. 2015). The geodetic parameter is a function of the angular spin velocity and of the mean density of the body. Depending on the value of that parameter and the accuracy of available observations, the Clairaut's equations may be developed to first, second or third order (Lanzano 1974). The boundary equations of the surface are written for axisymmetric bodies, thus we exclude bodies of triaxial shape in this study, like the dwarf planet Haumea. This method has been previously applied to the hydrostatic figures of Earth (Chambat et al. 2010) and Ceres (Rambaux et al. 2015; Park et al. 2016).

In the first part, we present the dataset used in this paper. Then, we recall the method to determine the equilibrium figure and the last section is dedicated to the description of the results.

\section{INPUT PARAMETERS}

Table 1 lists the five bodies with their physical properties (radii, bulk densities, rotation periods, and rotation ratios) determined by different sets of measurements (see the references in the Table). These TNOs are observed regularly by Earth-based and space telescopes in the infrared and visible wavelengths (e.g. Sheppard et al. 2008; Fornasier et al. 2013). Their rotational periods are determined through light curve measurements (Thirouin et al. 2010, 2014), and their shapes by stellar occultation (e.g. Roques et al. 2008). The mean densities of some dwarf planets and TNOs have been determined by using the orbital motion of their satellites or by fitting their shapes with a MacLaurin ellipsoid (i.e., assuming a uniform interior). Recently, Barr \& Schwamb (2016) suggested that large KBOs may be sorted into two groups: one presents a primordial composition with a mean density of about $1800 \mathrm{~kg} / \mathrm{m}^{3}$ whereas the second group has a larger density due to the loss of icy material via collisions. Here, we range possible interior models of our selected objects by assuming a simple two-layer structure: a silicate core and an icy shell with constant densities of 

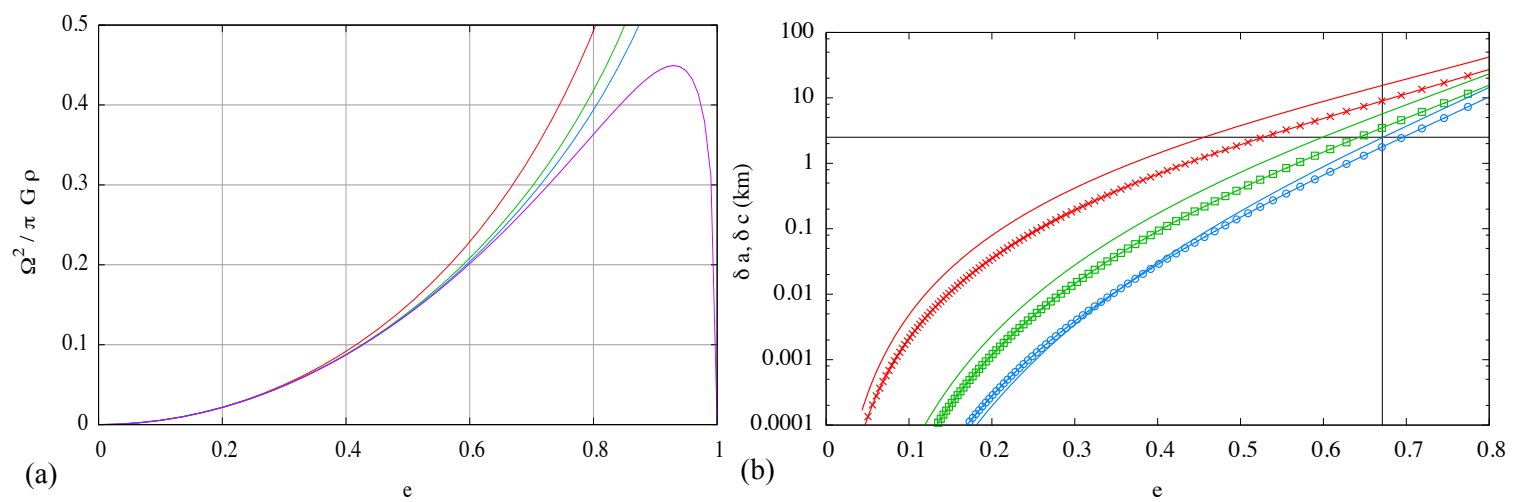

Figure 1. (a) Comparison between MacLaurin (violet) and the numerically computed solution at 1st (red), 2d (green) and 3rd (blue) order. The Jacobi ellipsoid case is expected for $\frac{\Omega^{2}}{\pi G \rho}=0.374$ but is not represented here. (b) Absolute difference between the equatorial radius $\delta a=a_{M L}-a_{3}$ (polar radius $\delta c$ ) computed from the MacLaurin equation and Clairaut's equations numerically solved at 1st (red), $2 \mathrm{~d}$ (green) and 3rd (blue) order. The results in $\delta a$ are plotted with lines whereas the results of $\delta c$ are represented with red cross line at first order, green square line at second order, and circle blue line at third order. The horizontal line represents $2.5 \mathrm{~km}$ whereas the vertical line represents $e=0.67$. The radius of the body is assumed to be $500 \mathrm{~km}$.

$\rho_{\text {sil }}=3000 \mathrm{~kg} / \mathrm{m}^{3}$ and $\rho_{\text {ice }}=920 \mathrm{~kg} / \mathrm{m}^{3}$ respectively, in agreement with the models of Barr \& Schwamb (2016). Then we express our results in terms of silicate mass fraction $\sigma$ corresponding to the mass of the core over the total mass:

$$
\sigma=\frac{\rho_{\text {sil }}\left(\rho-\rho_{\text {ice }}\right)}{\rho\left(\rho_{\text {sil }}-\rho_{\text {ice }}\right)}
$$

where $\rho$ is the TNO mean density.

\section{METHOD: HYDROSTATIC EQUILIBRIUM MODEL}

MacLaurin's ellipsoid is a shape solution for self-gravitating, rotating, and homogeneous bodies in hydrostatic equilibrium (e.g. Moritz 1990). The eccentricity of a MacLaurin ellipsoid is defined as $e^{2}=1-(c / a)^{2}$, with $a$ and $c$ the equatorial and polar radii, and is determined via (e.g. Chandrasekhar 1969)

$$
\frac{\Omega^{2}}{\pi G \rho}=\frac{2 \sqrt{1-e^{2}}}{e^{3}}\left(3-2 e^{2}\right) \arcsin e-6 \frac{1-e^{2}}{e^{2}},
$$

where $\Omega$ is the angular spin velocity, $G$ the gravitational constant, and $\rho$ the density.

The MacLaurin equation does not apply to stratified bodies, whose shapes are not an ellipsoid anymore (Hamy 1889; Moritz 1990). On the other hand, by comparing the Maclaurin solution with the one of the Clairaut's equations for a homogeneous body, we can explore the validity of the Clairaut solution as a function of the eccentricity. The Figure 1(a) shows the solutions for the MacLaurin and numerical Clairaut solutions for a homogeneous body. In order to determine the difference between the two solutions directly in the shape, we estimate the difference in the equatorial (and polar) radius for MacLaurin and numerical solution at order one, two, and three. These differences, quoted $\delta a$ (and $\delta c$ ), are represented in Figure 1(b) as a function of $e$. In this paper, we will require that $\delta a$ be smaller than 2.5 $\mathrm{km}$ to be sure to have a sufficient numerical precision with respect to the observationnal uncertainties. Indeed, this value is 10 times smaller than the uncertainty in equatorial radius from Braga-Ribas et al. (2013) (of $24 \mathrm{~km}$ ). It also corresponds to $e \leq 0.67$ (Figure 1(b)), and for that reason we truncate our results of the next section at that threshold.

The Clairaut's equations can be scaled by the mean spherical radius $R$ (i.e. Lanzano 1974; Chambat et al. 2010). Consequently, the quantity $\nu=(a-c) / R$, corresponding to the ratio between the equatorial and polar radii difference divided by the mean radius, is independent of $R$. The advantage of using $\nu$ is to be able to compare the solutions for TNOs selected in Table 1. 


\section{RESULTS AND DISCUSSION}

This section presents the equilibrium figures computed as a function of the rotation period $P$ and the silicate mass ratio $\sigma$ with comments for the five TNOs. The rotation period is ranged between 6 and 13 hours and the interval for the silicate mass ratio between 0 and 1 . An icy homogeneous body with a density of $920 \mathrm{~kg} / \mathrm{m}^{3}$ corresponds to $\sigma=0$ whereas the $\sigma=1$ case is for a purely silicate body with a density of $3000 \mathrm{~kg} / \mathrm{m}^{3}$. Note that the silicate mass ratio $\sigma$ is not a linear function of the mean density.

Figure 2 presents the differences $\delta \nu=\nu_{M L}-\nu_{3}$ between the homogeneous cases (index $M L$ for MacLaurin) and stratified cases computed to order three (index 3). We have superimposed in this Figure the data with uncertainties for the five bodies listed in Table 1. The white area located at the left corner of this figure is excluded, because it corresponds to bodies with an eccentricity larger than 0.67 for the stratified case (see section 3 ). The fast rotating and low density models present large signature of their stratification in the computed shapes and the maximum difference in $\delta \nu$ reaches a value of 0.108 . This value converted to $\delta(a-c)=(a-c)_{M L}-(a-c)_{3}$ is equal to $54 \mathrm{~km}$ for a $500 \mathrm{~km}$ radius body.

The limit $\delta \nu=0.07$, or $\delta(a-c)=35 \mathrm{~km}$ for a $500 \mathrm{~km}$ radius body, is on a par with the current level of groundbased observational uncertainties taking $25 \mathrm{~km}$ for the equatorial radius uncertainty (e.g. Braga-Ribas et al. 2013) and assuming the same value for the polar radius uncertainty. Consequently, all bodies above that curve i.e. $\delta \nu \geqslant 0.07$ (dark red and black colors) would potentially present a detectable signature of internal stratification with current state-of-art measurements.

In addition, similar mapping in period $P$ and silicate mass ratio $\sigma$, excluding the area where $e>0.67$, provide an estimation of the impact of the order on the solution. The difference between second and first order $\nu_{2}-\nu_{1}$ is at maximum 0.03 or $(a-c)_{2}-(a-c)_{1}=15 \mathrm{~km}$, where the index represents the order in the Clairaut's equations. The difference between third and second order, $\nu_{3}-\nu_{2}$, reaches 0.01 or $5 \mathrm{~km}$ when converted into $(a-c)_{3}-(a-c)_{2}$. These values show the necessity to use the second and third order development for the solutions located at the boundary at the left corner maps of Figure 2 for accuracy about $2.5 \mathrm{~km}$. Below we describe the results specific to each of the five bodies in Table 1 and Figures 2, 3, and 4. The last Figure represents the $(a-c)$ for each body as function of the mass silicate ice ratio $\sigma$ for numerically integrated Clairaut's equations and MacLaurin solutions.

Salacia has been observed by the Spitzer Space Telescope and Herschel Space Observatory (e.g. Fornasier et al. 2013). Through these observations and thermal modeling, its radius has been inferred as $427 \pm 22.5 \mathrm{~km}$ (Fornasier et al. 2013) and by combining with orbital tracking of its companion Actea, its density has been derived as $1290_{-230}^{+290}$ $\mathrm{kg} / \mathrm{m}^{3}$ (Fornasier et al. 2013) or $\sigma=0.41 \pm 0.20$. Due to its fast rotation period of 6.5 hours (Thirouin et al. 2014), Salacia is the most oblate body in our data selection. According to the Figures 3 and 4 , the radi difference $(a-c)$ of Salacia is between $67 \mathrm{~km}$ and upper to $126 \mathrm{~km}$ because it falls in the excluded zone where the eccentricity is larger than 0.67. According to the Figure 2 the maximum $\delta \nu=0.1$ for Salacia is reached at $\sigma=0.33$ and it converts into 43 $\mathrm{km}$ in $\delta(a-c)$ (Fig. 4). In practice an object of Salacia's size is expected to contain some porosity, as suggested by the relatively low density (the lowest among the bodies considered here). Hence our simplified two-layer model has to be used with caution in this case.

The radius of Makemake has been measured by stellar occultation to $715 \pm 3.5 \mathrm{~km}$ (Ortiz et al. 2012; Brown 2013) and its rotational period is $7.771 \pm 0.003$ hours (Heinze \& de Lahunta 2009). Recently, Parker et al. (2016) discovered a moon orbiting Makemake but its tracking is too sparse for the derivation of Makemake's mass. Consequently, there is no observatonal constraints on $\sigma$ for Makemake and we explore it from 0 to 1 . The maximum value in $\nu$ for Makemake is 0.27 and it corresponds to $(a-c)$ equal to $193 \mathrm{~km}$ with a mean radius of $715 \mathrm{~km}$ (Fig. 3 and 4). It is obtained for $\sigma=0.035$ due to the 0.67 limit in eccentricity. The minimum radii difference $(a-c)$ is obtained for $\sigma=1$ and it is equal to $56 \mathrm{~km}$ (Fig. 4). As shown in Figure 4 Makemake presents the larger radii difference of the selected TNOs and the largest departure between the MacLaurin to two-layer solutions wich reaches $40 \mathrm{~km}$ for $\sigma=0.37$.

The rotation period of Quaoar has been determined equal to 17.68 hours (Ortiz et al. 2003) and revised recently to $8.839 \pm 0.003$ hours (Thirouin et al. 2010; Rabinowitz et al. 2007), which is the preferred value of Fornasier et al. (2013). The radius of Quaoar measured with Herschel Space Observatory instruments and thermal modeling is $535 \pm$ $19 \mathrm{~km}$ and the density estimated by using the mass of Fraser et al. (2013) is $2180_{-360}^{+430} \mathrm{~kg} \mathrm{~m}^{-3}$ (Fornasier et al. 2013). Quaoar's shape has also been determined by stellar occultation observations (Braga-Ribas et al. 2013), which led to a density of $1990 \pm 460 \mathrm{~kg} \mathrm{~m}^{-3}$ by fitting the measurements with a MacLaurin ellipsoid. Figures 2, 3, and 4 show the solution for Quaoar corresponding to the stellar occultation observations. In this case, for two-layer models, the $(a-c)$ values vary between 34 and $45 \mathrm{~km}$. The difference with the MacLaurin solution reaches $19 \mathrm{~km}$ for $\sigma=0.621$. 


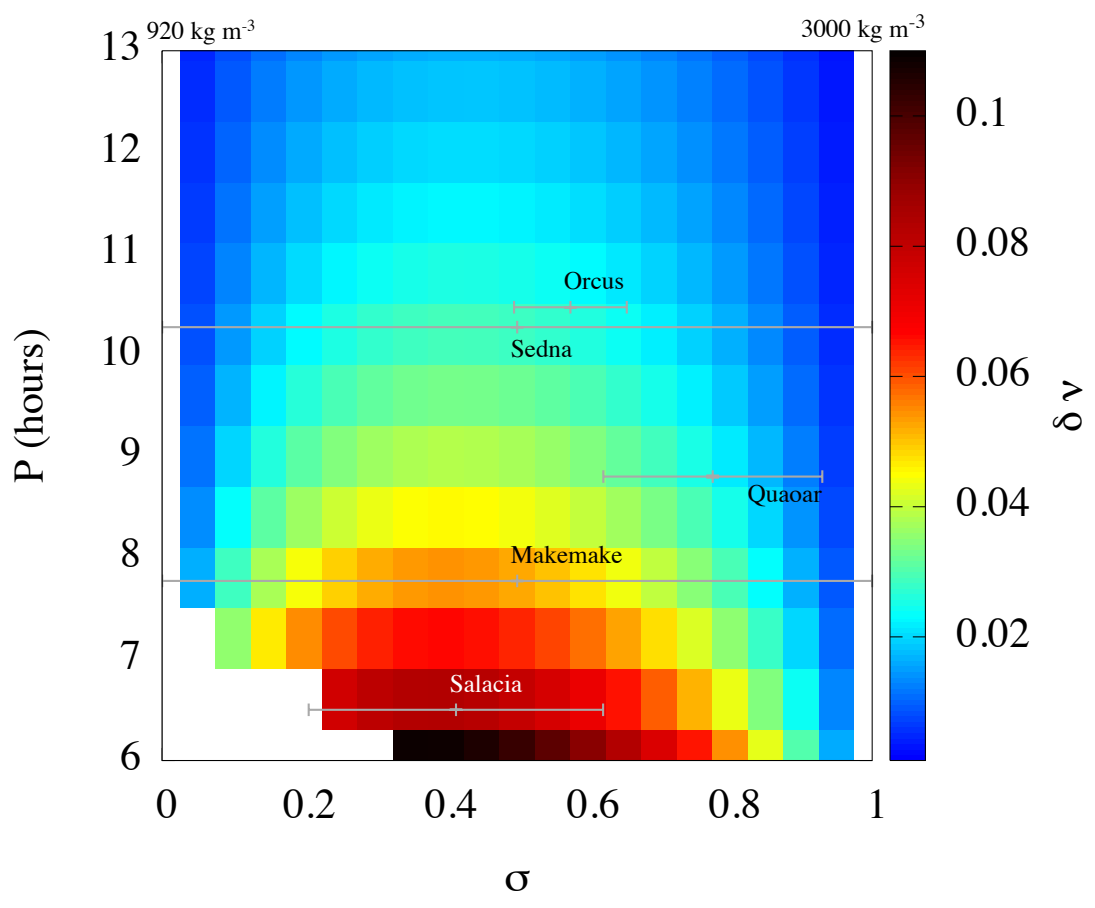

Figure 2. Difference $\delta \nu=\nu_{M L}-\nu_{3}=(a-c)_{M L} / R-(a-c)_{3} / R$ computed for the homogeneous case (MacLaurin ML) and a two layer model, developed to order 3 . The horizontal lines represent the uncertainties in the mean densities. The error bars on the rotation periods are too small to be legible on this plot. $\sigma=0$ corresponds to a homogeneous body of density equal to $920 \mathrm{~kg} / \mathrm{m}^{3}$ whereas $\sigma=1$ corresponds to a homogeneous body of density $3000 \mathrm{~kg} / \mathrm{m}^{3}$.

This value is about two times smaller than the uncertainty obtained by stellar occultation equal to $35 \mathrm{~km}$ using the values deduced from Braga-Ribas et al. (2013).

Sedna's orbit has a semi-major axis of 515.5 AU and an orbital eccentricity of 0.85 . No satellite has been detected so far, therefore Sedna's mean density is unknown. Its rotation period is 10.273 hours (Gaudi et al. 2005) and the $\delta \nu$ reaches the maximum value of 0.0286 with a $\sigma$ of 0.4 . The resulting difference in $\delta(a-c)$ between the homogeneous and stratified cases may reach $14 \mathrm{~km}$ with a $(a-c)$ for stratified model equal to $40 \mathrm{~km}$.

The surface of Orcus and its low density (Fornasier et al. 2013) suggest that this TNO contains a significant fraction of water. Orcus rotates in 10.47 hours (Thirouin et al. 2010). According to Figures 3 and 4 the $\delta(a-c)$ of Orcus is in the range $25-31 \mathrm{~km}$ and this value is almost $10 \mathrm{~km}$ below the MacLaurin solutions.

In this paper, the $\nu$ diagnostic on the stratification of TNOs is computed by assuming that the bodies shape corresponds to an hydrostatic equilibrium shape. However, TNOs may present some topography related to mountains or craters. These two features have been discussed in details in Braga-Ribas et al. (2013) for Quaoar case and we extend the discussion for the five selected TNOs. The height at which a mountain can be supported at the surface of a TNO depends inversely on the square density and radius of the body (Johnson \& McGetchin 1973). By assuming an ultimate strength of $3.03 \mathrm{MPa}$ for the ice and that the failure occurs for a surface strength equal to two times the strength of the material (see Johnson \& McGetchin 1973), then the topography height is below $10 \mathrm{~km}$ for Makemake, Quaoar and Sedna but it is about 20 and $31 \mathrm{~km}$ for Orcus and Salacia. For these last two bodies the size of the mountains is able to perturb the interpretation of the data and has to be taken into account.

The craters result from the collisional environment of the TNOs that are thought to occur regularly at rate of hundred million years (see Barr \& Schwamb 2016). The depth of the craters are scaled with the radius of the body and by using the scaling law of Leliwa-Kopystyński et al. (2008) the depth of crater is roughly 0.3 body radius for the limit before disruption and by assuming that the surface is made of $\mathrm{H}_{2} 0$-ice (see also Braga-Ribas et al. 2013). In this critical case the crater depth can be deep as $215 \mathrm{~km}$ for Makemake and then between 160-130 km for Quaoar, Sedna, Orcus, and Salacia. The presence of such deep craters may also perturbed the interpretation of the shape in terms of hydrostatic equilibrium shape. 


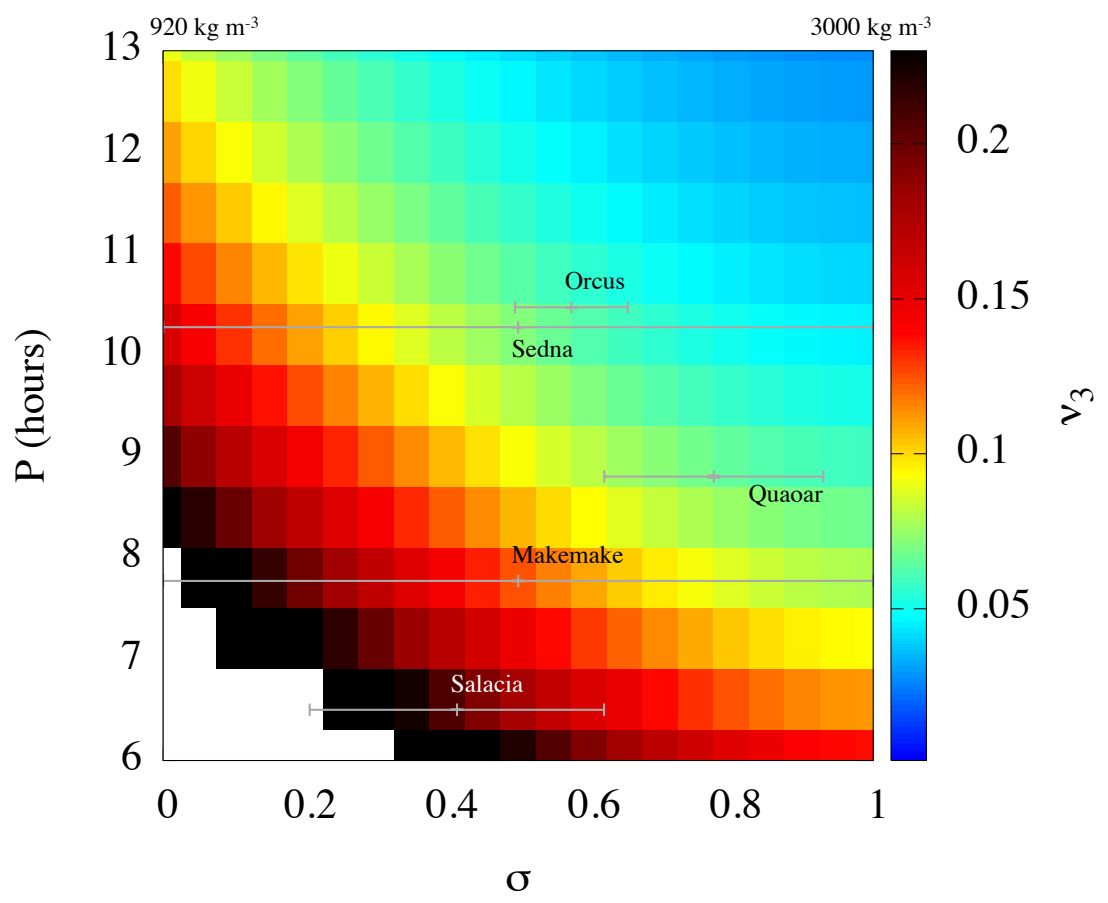

Figure 3. Solution of $\nu_{3}=(a-c)_{3} / R$ computed for the two layer model, developed to order 3 , for the parametric space covered in this study and for specific bodies. $\sigma=0$ corresponds to a homogeneous body of density equal to $920 \mathrm{~kg} / \mathrm{m}^{3}$ whereas $\sigma=1$ corresponds to a homogeneous body of density $3000 \mathrm{~kg} / \mathrm{m}^{3}$.

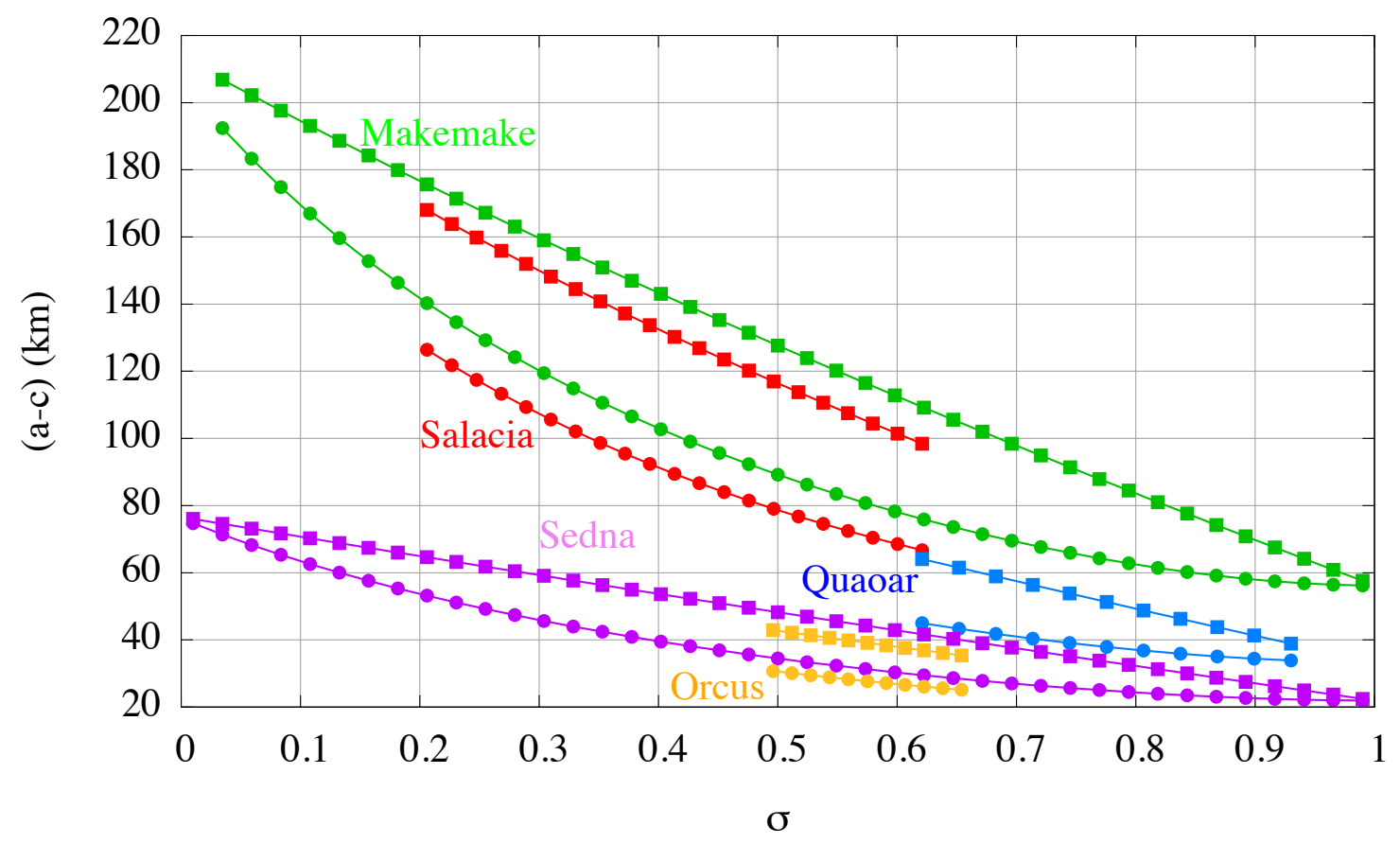

Figure 4. Solutions of the five selected TNOs (see Table 1) for $(a-c)$ as a function of the mass silicate ratio $\sigma$. The line with circles represents the heterogeneous solutions computed from Clairaut's equations and the line with squares are plotted for the MacLaurin solutions. The two sets of solutions are close at $\sigma=0$ and 1 as expected (see Fig. 1(a) for an estimation of the differences). The figure shows that the difference between the homogenous and stratified solutions can be larger than the observation uncertainties, at least for Makemake and Salacia. 


\section{CONCLUSION}

The accuracy of stellar occultation observations has significantly improved over the past decades and now reaches about twenty kilometer level for distant TNOs (e.g. Braga-Ribas et al. 2013). In preparation for the interpretation of future observations in terms of interior structure, it is important to develop accurate shape models. As many of the dwarf planets and TNOs are fast rotators, the development of the Clairaut's equations to order two is required to properly represent the shapes of these bodies and even to order three for the very fast rotators and low densities. An alternative method based on numerical nonperturbative development is also available to compute the structure of 2-layer spheroids (Hubbard 2013) (see sec. 3 of the present paper). The TNO Makemake and Salacia may be good candidates for the application of this method since the difference in $\delta(a-c)$ between a homogeneous and a stratified model reaches 40 and $43 \mathrm{~km}$, respectively. Quaoar is also potentially a good candidate with $\delta(a-c)$ of $16 \mathrm{~km}$. This value is smaller than the $35 \mathrm{~km}$ derived from (Braga-Ribas et al. 2013) but future measurements shall improve its shape. The caveat of the method is to suppose hydrostatic equilibrium: for small radius body, mountains and large craters may reach values of the order of $(a-c)$ and interfere the interpretation of shape observations. Several predictions of stellar occultations, notably for Quaoar, are planned ${ }^{1}$ and with future DR2 Gaia stellar catalogue and better orbit determination models (Desmars et al. 2015) these predictions will be increasingly accurate allowing more measurements and better determination of Quaoar and TNOs' shapes in general.

Part of this work has been carried out at the Jet Propulsion Laboratory, California Institute of Technology, under contract to NASA.

\section{REFERENCES}

Barr, A. C., \& Schwamb, M. E. 2016, MNRAS, 460, 1542

Braga-Ribas, F., Sicardy, B., Ortiz, J. L., et al. 2013, ApJ, 773,26

Brown, M. E. 2013, ApJL, 767, L7

Chambat, F., Ricard, Y., \& Valette, B. 2010, Geophysical Journal International, 183, 727

Chandrasekhar, S. 1969, Ellipsoidal figures of equilibrium

(The Silliman Foundation Lectures, New Haven: Yale

University Press, 1969)

Desmars, J., Camargo, J. I. B., Braga-Ribas, F., et al. 2015, A\&A, 584, A96

Fornasier, S., Lellouch, E., Müller, T., et al. 2013, A\&A, 555, A15

Fraser, W. C., Batygin, K., Brown, M. E., \& Bouchez, A. 2013, Icarus, 222, 357

Gaudi, B. S., Stanek, K. Z., Hartman, J. D., Holman, M. J., \& McLeod, B. A. 2005, ApJL, 629, L49

Hamy, M. 1889, Annales de l'Observatoire de Paris, 19, F1

Heinze, A. N., \& de Lahunta, D. 2009, AJ, 138, 428

Hubbard, W. B. 2013, ApJ, 768, 43

Johnson, T. V., \& McGetchin, T. R. 1973, Icarus, 18, 612

Lanzano, P. 1974, Astrophysics and Space Science, 29, 161

Leliwa-Kopystyński, J., Burchell, M. J., \& Lowen, D. 2008, Icarus, 195, 817
Moritz, H. 1990, The figure of the Earth : theoretical geodesy and the Earth's interior (Karlsruhe : Wichmann, c1990.)

Ortiz, J. L., Gutiérrez, P. J., Casanova, V., \& Sota, A. 2003, A\&A, 407, 1149

Ortiz, J. L., Sicardy, B., Braga-Ribas, F., et al. 2012, Nature, 491, 566

Park, R. S., Konopliv, A. S., Bills, B. G., et al. 2016, Nature, 537, 515

Parker, A. H., Buie, M. W., Grundy, W. M., \& Noll, K. S. 2016, ApJL, 825, L9

Rabinowitz, D. L., Schaefer, B. E., \& Tourtellotte, S. W. 2007, AJ, 133, 26

Rambaux, N., Chambat, F., \& Castillo-Rogez, J. C. 2015, A\&A, 584, A127

Roques, F., Georgevits, G., \& Doressoundiram, A. 2008, The Kuiper Belt Explored by Serendipitous Stellar Occultations, ed. M. A. Barucci, H. Boehnhardt, D. P. Cruikshank, A. Morbidelli, \& R. Dotson, 545-556

Sheppard, S. S., Lacerda, P., \& Ortiz, J. L. 2008, Photometric Lightcurves of Transneptunian Objects and Centaurs: Rotations, Shapes, and Densities, ed. M. A. Barucci, H. Boehnhardt, D. P. Cruikshank,

A. Morbidelli, \& R. Dotson, 129-142

Tancredi, G., \& Favre, S. 2008, Icarus, 195, 851

Thirouin, A., Noll, K. S., Ortiz, J. L., \& Morales, N. 2014,

${ }^{1}$ see the website http://lesia.obspm.fr/lucky-star/predictions/index2017.AtdenA, 569, A3 
Thirouin, A., Ortiz, J. L., Duffard, R., et al. 2010, A\&A, 522, A93 